\title{
TREINAMENTO DE FORÇA EM CRIANÇAS: SEGURANÇA, BENEFÍCIOS E RECOMENDAÇÕES
}

\author{
Cristiano Cavedon Ughini, Universidade Federal do Rio Grande do Sul - UFRGS, Porto \\ Alegre, Rio Grande do Sul - Brasil \\ Cristiano Becker, Universidade Federal do Rio Grande do Sul - UFRGS, Porto Alegre, Rio \\ Grande do Sul - Brasil \\ Ronei Silveira Pinto, Universidade Federal do Rio Grande do Sul - UFRGS, Porto Alegre, \\ Rio Grande do Sul - Brasil
}

\begin{abstract}
RESUMO
O treinamento de força (TF) - também denominado treinamento com pesos - refere-se a um método especializado de condicionamento físico que envolve o uso progressivo de cargas resistivas desenvolvidas para aumentar ou manter a aptidão muscular. Atualmente o número de jovens engajados em programas de treinamento que incluem o TF está crescendo de forma vertiginosa. Tendo isso em vista, o objetivo dessa presente revisão é relatar os principais aspectos relacionados à segurança e à eficácia do $\mathrm{TF}$ em crianças e jovens e apresentar linhas de orientação desenvolvidas especificamente para essa população. O método utilizado foi uma revisão sistemática com a localização de artigos publicados entre 1993 e 2001 nas bases de dados PubMed, Scielo e Scopus. As evidências científicas encontradas nos estudos analisados indicam que o TF é saudável e apresenta diversos benefícios para crianças e adolescentes quando esses são instruídos, apropriadamente, por instrutores qualificados que se baseiam em linhas de orientação corretas de treino. Assim sendo, o TF pode ser praticado por crianças, pois se apresenta de forma segura e benéfica à saúde dessa população.
\end{abstract}

Palavras-Chave: Treinamento de força; Crianças; Jovens; Linhas de orientação; Lesão.

\section{YOUTH STRENGTH TRAINING: SAFETY, BENEFITS AND GUIDELINES}

\begin{abstract}
Strength training (ST) - also named as weight training - refers to a specialized method of physical training that involves the progressive use of resistive loads developed to increase or maintain the physical fitness. Nowadays the number of youth engaged in physical programs that includes the ST is increasing quickly. In view of that, the aim of this review is report the main aspects related to youth ST safety, efficacy, and guidelines developed especially for this sort of population. The used method was a systematic review with papers published between 1993 and 2011, in PubMed, Scielo and Scopus. The scientific evidence found in the assessed studies indicates that ST is health and has many benefits for children and adolescents when these are instructed properly by qualified instructors who are based on proper guidelines training. Thus, the ST can be practiced by youth, as it is safe and beneficial to the health of this sort of population.
\end{abstract}

Key-Words: Strength training; Children; Youth; Guidelines; Injury. 


\section{INTRODUÇÃO}

Atualmente o número de jovens engajados em programas de treino que incluem o treinamento de força (TF) está crescendo de forma vertiginosa. Todavia, apesar de muitos profissionais da área da saúde terem considerado o $\mathrm{TF}$ inseguro e potencialmente prejudicial para o desenvolvimento do sistema musculoesquelético, evidências que têm relatado a segurança e eficácia do TF para crianças e adolescentes aumentaram consideravelmente na última década. ${ }^{1-3}$ Além disso, programas de treinamento supervisionados e adequadamente planejados estão cada vez mais aceitos e difundidos por vários profissionais de saúde. ${ }^{4-5}$

Há, entretanto, um substancial interesse e preocupação dos profissionais de educação física para com a segurança e adequação das crianças e adolescentes que participam de programas de treinamento, que englobem algum tipo de TF. Esse fato está vinculado, principalmente, a estudos atuais, que têm recomendado exercícios de força para reduzir o percentual de lesões e aumentar o rendimento de indivíduos desta faixa etária. ${ }^{1}$

Percebendo esses aspectos, devido à crescente popularidade do TF entre crianças e adolescentes, surge uma constante preocupação sobre a participação dos mesmos nessa atividade. Portanto, a presente revisão tem como objetivo relatar os principais aspectos relacionados à segurança, aos benefícios, e às recomendações do TF em crianças e jovens.

\section{MÉTODOS}

Foi desenvolvido o seguinte método para o levantamento da literatura correspondente: tipos de estudos - ensaios clínicos prospectivos e randomizados, artigos e livros publicados em datas compreendidas de 1993 a 2011, que apresentaram informações relevantes e referentes à 
discussão em questão; estratégia de busca para identificação dos estudos: utilização de meios eletrônicos, incluindo as bases de dados Scopus, Scielo e Pubmed.

A busca foi realizada nas línguas inglesa e portuguesa. Somente estudos passíveis de serem obtidos em sua íntegra foram analisados. Para isso, foram utilizadas as seguintes palavraschave, por lógica booleana (palavras combinadas por “AND”): treinamento de força (strength training), criança (child), recomendações (guidelines) e lesão (injury).

Todos os resumos obtidos por meio das diferentes estratégias de busca foram avaliados e classificados em:

a) elegíveis: estudos que apresentam relevância e têm possibilidade de ser incluídos na revisão;

b) não elegíveis: estudos sem relevância, sem possibilidade de inclusão na revisão sistemática.

A relevância deste artigo consiste na possibilidade de proporcionar aos profissionais da área da educação física os subsídios teóricos sobre o treinamento de força realizado com crianças, salientando-se que é imprescindível o conhecimento de tais informações para o desenvolvimento de intervenções qualificadas com o exercício físico.

\section{FUNDAMENTAÇÃO TEÓRICA}

\section{Definição}

O termo "treinamento de força" - também denominado treinamento com pesos - referese a um método especializado de condicionamento físico que envolve o uso progressivo de cargas resistivas com o objetivo de aumentar ou manter a aptidão muscular. ${ }^{4}$ Esses programas 
podem envolver o uso de pesos livres, equipamentos, bandas elásticas ou o uso do peso corporal do próprio praticante. A quantidade e a forma de resistência utilizada e a frequência dos exercícios de força são determinados pelos diferentes objetivos do programa. ${ }^{6}$

O TF deve ser distinguido dos esportes competitivos de halterofilismo e levantamento de peso em que os indivíduos diariamente treinam de forma intensa para maximizar o tamanho muscular, força e potência. ${ }^{4}$

Ainda que o TF aconteça em lugares comuns como nas escolas, centros recreativos e campos esportivos, tanto para meninos como meninas, a preocupação sobre a segurança e eficácia do TF ainda persiste.

\section{Segurança relativa no treinamento de força em jovens}

Embora o TF em jovens possa envolver algum risco inerente de lesões musculoesqueléticas, esse risco não parece ser maior do que outros esportes e atividades recreacionais em que crianças e adolescentes participam regularmente. Alguns estudos mostram que o treinamento de força apresenta um índice de lesão muito baixo quando comparado a outros esportes escolares. ${ }^{7}$ Esses dados mostram que, se realizado de maneira correta, com o auxílio de um profissional qualificado, o TF pode ser considerado uma prática bastante segura. Como suporte dessa observação, dados recentes indicam que o futebol americano teve a taxa mais alta de lesões dentre as nove atividades estudadas, dentre elas, o TF. ${ }^{8}$

Outro método de TF - o treinamento pliométrico - também pode ser um método relativamente efetivo e seguro no condicionamento de crianças e adolescentes se prescrito apropriada e progressivamente ao longo do tempo. Marginson et al. ${ }^{9}$ compararam os sintomas do dano 
muscular induzido pelo exercício pliométrico entre adultos e crianças, mostrando que, embora ambos os grupos tenham apresentado danos, esses foram bem menos severos no segundo grupo. Esses resultados, segundo os autores, podem incluir maior flexibilidade, evitando um excessivo alongamento dos sarcômeros na fase excêntrica, menor número de fibras de contração rápida e, talvez, um estilo de vida mais ativo. A adição de qualquer tipo de TF deve ser cuidadosamente considerada para a dose total de exercício de atletas jovens e como esse tipo de treinamento poderá aumentar o estresse crônico repetitivo relacionado ao desenvolvimento do sistema musculoesquelético.

As lesões nesse tipo de treinamento podem acontecer se a intensidade, o volume ou a freqüência do programa excederem a habilidade dos participantes de realizar corretamente os movimentos ou de se recuperar de treinamentos anteriores. Um menino de doze anos, por exemplo, desenvolveu rabdomiólise após executar repetições excessivas (mais de 250) de saltos sem contra-movimento como punição por conversar em uma aula de educação física escolar. $^{10}$

\section{Lesões}

Durante muito tempo, o TF para crianças não era recomendado por presumir que este levava a alto risco de lesões. Hoje, sabe-se que, geralmente, o risco de lesões associadas a este tipo de treinamento é semelhante em crianças e adultos.

Estudos recentes indicam um risco relativamente baixo de lesões em crianças e adolescentes que seguiram adequadamente a metodologia correta de acordo com a idade, e que tiveram uma qualificada supervisão e instrução ao longo do $\mathrm{TF} .{ }^{7}$ Uma grande variedade de programas de TF que alternaram desde sessões de uma série em equipamentos adaptados, até sessões de 
múltiplas séries usando diferentes equipamentos, se apresentou seguro e eficaz para o público em questão. Além disso, esses achados são sustentados por outros estudos que mostram que a modalidade de levantamento de peso pode também ser segura para crianças e adolescentes, desde que, supervisionadas por treinadores bem esclarecidos que acompanhem todas as sessões de treino e competições para conseguirem uma correta e cautelosa prescrição da carga a ser levantada. ${ }^{1}$

Recentemente foram examinados dados sobre a incidência de lesões relacionadas ao TF nas salas de emergências dos Estados Unidos da América. ${ }^{11}$ Foi encontrada uma correlação negativa entre o aumento da idade e o número de lesões acidentais. Assim sendo, à medida que a idade do grupo crescia $(8-13,14-18,19-22$ e 23-30) o número de lesões acidentais decrescia significativamente. Como exemplo, foi notado que dois terços das lesões entre 8-13 anos ocorreram nas mãos ou nos pés, e eram constantemente descritas como "beliscadas" e “deixar cair” (Figura 1). Contrariamente, o número de torções articulares e distenções musculares foram maiores nos grupos mais velhos, mostrando assim que as lesões ocorridas nas idades mais jovens ocorrem por desatenções e acidentes, e não por lesões crônicas do treino.

Coutts et al. ${ }^{2}$ acompanharam 21 adolescentes com uma média de 16,7 anos que realizavam TF três vezes por semana, realizando, aproximadamente, oito exercícios por sessão. Nesse estudo, não foi encontrado nenhum relato de lesão por um período de treino de aproximadamente 12 semanas. 


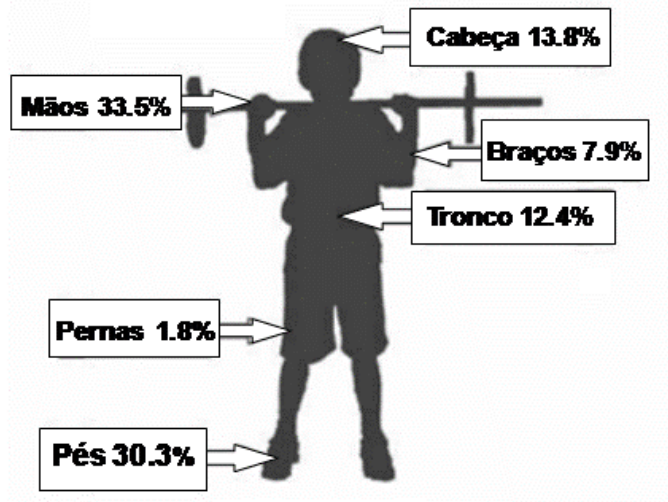

$8-13$ anos

$77.2 \%$ Acidental

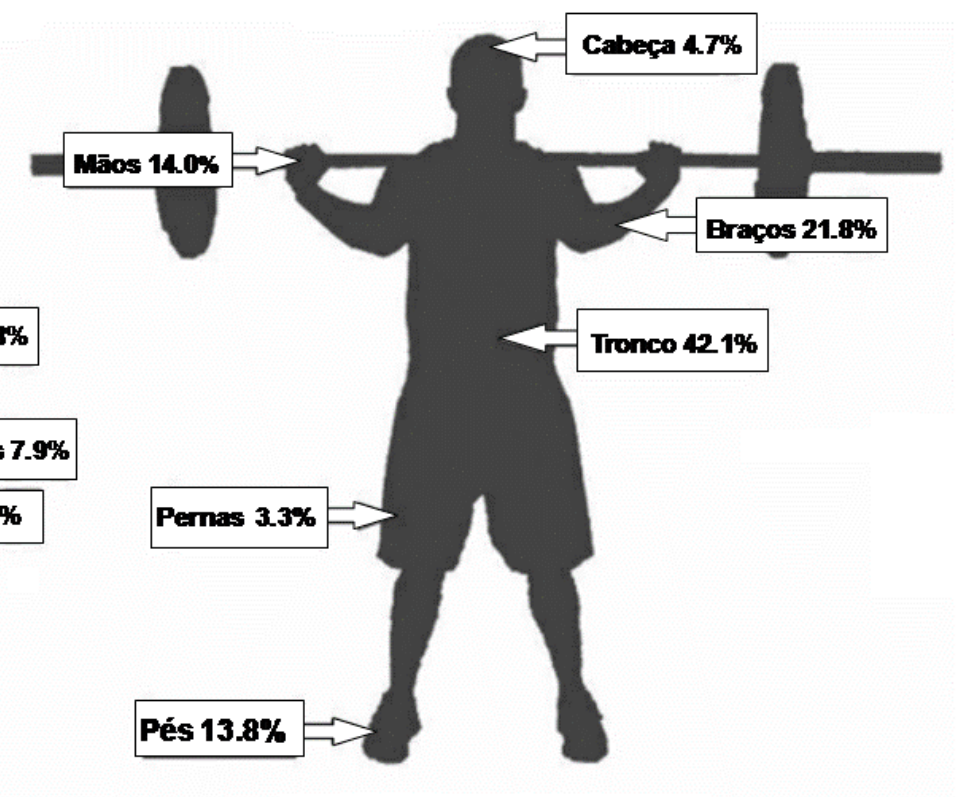

$23-30$ anos

\section{5\% Acidental}

Figura 1 - Percentual de lesões das categorias mais jovens e mais velhas Fonte: Adaptado de KERR.. ${ }^{11}$

Lillegard et al. ${ }^{12}$ acompanharam 52 adolescentes que realizaram TF por dez semanas, com uma frequência de três vezes por semana. Nesse estudo, durante o período de treinamento, ocorreu somente uma lesão, todavia sem gravidade e descrita como sendo acidental.

Por sua vez Faigenbaum e colaboradores ${ }^{13}$ observaram 56 crianças dentre meninos e meninas (média de 9,3 anos) durante oito semanas nas quais estas realizavam TF uma ou duas vezes semanais e não relataram nenhuma ocorrência de lesão.

Faigenbaum et al. ${ }^{14}$ acompanharam novamente 41 crianças dentre meninos e meninas com médias de idade variando de 8 a 12 anos de idade. Essas crianças, por sua vez, realizaram TF 
duas vezes por semana, durante oito semanas. E, novamente, não foi observada nenhuma lesão durante o período avaliado.

Em outro estudo, no qual o autor avaliou a taxa de lesão em adolescentes, foi observado que o TF e o levantamento de peso são mais seguros quando comparados a vários outros esportes e atividades em questão, ${ }^{7}$ explicitado na Tabela 1 . Nesse estudo, a taxa de lesão a cada 100 horas de participação foi de 0,80 para Rúgbi e 0,014 e 0,0035 para futebol e TF, respectivamente, mostrando, assim, que o TF quando realizado corretamente e com supervisão apresenta baixo risco de lesão.

Tabela 1 - Taxa de lesão a cada 100 horas de participação em diferentes modalidades

\begin{tabular}{lr}
\hline \multicolumn{1}{c}{ ESPORTES } & \multicolumn{1}{c}{ TAXA DE LESÃO } \\
(lesão.1000horas & -1) \\
\hline Rugby & 0,800 \\
Futebol & 0,014 \\
TF & 0,0035 \\
\hline
\end{tabular}

TF: Treinamento de força.

Fonte: Adaptado de HAMILL. ${ }^{7}$

\section{Fatores de risco relacionados ao treinamento de força}

De maneira geral, se os programas de TF são conduzidos por profissionais qualificados e são planejados de acordo com a idade, o risco de lesão articular é insignificante. ${ }^{15}$ Técnicos, professores e treinadores devem, portanto, sempre estar atentos do risco inerente associado com o TF e devem se preocupar em diminuí-los seguindo uma linha de orientação préestabelecida. Os fatores de risco que estão comumente associados às lesões no esporte em jovens são: estirão de crescimento, idade, maturidade biológica, tamanho corporal, técnico desqualificado e lesões anteriores. Outros fatores hipotetizados, mas ainda não testados são: 
baixo condicionamento, desequilíbrio muscular, nutrição inadequada, equipamento impróprio, técnica de exercício pobre e erros de treinamento. ${ }^{16}$

Com uma instrução qualificada, uma progressão gradual do treinamento e conhecimento do desenvolvimento das crianças e dos adolescentes, os jovens podem ficar mais fortes, se sentirem bem a respeito de seu desempenho e se divertirem. ${ }^{4}$ Com esse programa apropriado, não ocorrerá influência sobre o crescimento linear, sobre as placas de crescimento ou sobre o sistema cardiovascular, ainda que se deva ter um cuidado maior com alunos que apresentam hipertensão arterial, pois eles podem necessitar de medicamentos para reduzir o potencial de aumento da pressão arterial com o TF. ${ }^{6}$

Cuidado especial também deve ser dado às crianças com sobrepeso. Embora pareçam mais fortes, em detrimento de seu tamanho, geralmente apresentam um mau condicionamento devendo ser submetidos a mesma supervisão e roteiros que outras populações.

\section{Treinamento de força como fator preventivo de lesões}

Estudos prévios demonstram, em crianças e adolescentes, um decréscimo de incidências de lesões em indivíduos que participaram de programas de $\mathrm{TF} .{ }^{4}$ Isso foi evidenciado em atletas escolares de futebol americano e nadadores, que, em suas pré-temporadas, seus treinadores incluíram programas com TF, verificando um decréscimo da taxa de lesão de seus respectivos atletas. ${ }^{1}$

Ainda avaliando indivíduos escolares atletas de ambos os sexos, foi visto que a taxa de lesão para aqueles que realizavam TF era de $26,2 \%$ comparado aos $72,4 \%$ para aqueles que não o 
praticavam. Além disso, o tempo requerido para a reabilitação dos atletas que tiveram algum tipo de lesão era de apenas 2,02 dias para o primeiro grupo e de 4,82 dias para o segundo grupo. ${ }^{1}$

Em determinada linha de estudo, o implemento de um programa de TF de um ano, mostrou-se efetivo no decréscimo de incidências de lesões em jogadores escolares de futebol e na eliminação do desequilíbrio articular em jogadores colegiais de futebol americano, diminuindo a reincidência de lesões tendíneas. ${ }^{17}$

$\mathrm{Na}$ verdade, os mais variados programas de treinamento que incluam em suas rotinas exercícios de força mostram-se efetivos na estratégia de reduzir as lesões decorrentes do esporte de crianças/adolescentes atletas. ${ }^{1,3}$ Todavia, alguns dos artigos citados acabam utilizando-se de múltiplas intervenções no intuito de diminuir o risco de lesão do indivíduo. Assim sendo, algumas vezes, torna-se difícil julgar qual dos inúmeros aspectos das variadas intervenções é que geraram tal benefício.

Um dos mecanismos pelo qual o TF pode prevenir e diminuir o risco de lesões inclui o aumento de força de estruturas de sustentação, como ligamentos, tendões e ossos; o aumento da habilidade que um músculo treinado tem de obter mais energia antes da fadiga, retardandoa; e o desenvolvimento do equilíbrio de determinado grupo muscular que envolve e sustenta uma articulação específica. ${ }^{18}$

Portanto, o TF pode diminuir a incidência de lesões em jovens atletas, tanto pelo aumento da força músculo-esquelética quanto pelo desenvolvimento do equilíbrio muscular de determinado grupo muscular que envolve e sustenta uma articulação específica. Isso foi 
observado por diminuição das taxas de lesões, em adolescentes, que participaram de programas de TF na pré-temporada de seus respectivos esportes.

\section{Benefícios relacionados à saúde}

Além de aumentar a força muscular dos jovens, a participação regular em um TF tem o potencial de influenciar outras medidas de saúde e condicionamento físico. Até o presente, alguns dados sugerem que o TF para jovens pode alterar favoravelmente parâmetros de saúde e aumentar o desempenho no esporte e nas habilidades motoras. Tem a capacidade, ainda, de reduzir lesões. ${ }^{19}$

Algumas rotinas de treinamento que englobem algum tipo de $\mathrm{TF}$ (com correta instrução da técnica) têm mostrado em crianças/adolescentes atletas uma melhora biomecânica do movimento, melhora das habilidades funcionais e redução do número de lesões relacionadas ao esporte. ${ }^{1}$ Alguns programas de TF para crianças ainda permitem dúvidas quanto à eficácia e segurança. Entretanto em programas devidamente prescritos e competentemente supervisionados têm-se observado melhora nas variáveis relacionadas à saúde.

Em crianças obesas, o TF tem sido incorporado como estratégia de controle de peso para essa população, a fim de acelerar o processo metabólico sem alto impacto e auxiliar na redução de gordura. ${ }^{1,19}$ Ademais, um estudo de Lau et al. ${ }^{20}$ mostrou que o TF aumenta a sensibilidade do hormônio leptina em adolescentes obesos.

Influências positivas do TF também têm sido encontradas em relação à capacidade de produção de força máxima, ao aumento da densidade mineral óssea e à resposta metabólica e composição corporal de crianças e adolescentes. ${ }^{19-22} \mathrm{O}$ bem-estar psicológico também tem 
recebido bastante importância como resposta ao TF em jovens. De acordo com Faigenbaum, ${ }^{23}$ uma vez que um programa de TF seja bem planejado e supervisionado por profissionais bem informados sobre aspectos relacionados com a infância e a adolescência, o mesmo pode oferecer benefícios psicossociais comparáveis a outras atividades e esportes. Em que pese exista poucos estudos investigando o assunto, parece que os benefícios do TF sobre o bem estar psicossocial irão depender da intensidade, da freqüência e da duração do treinamento, bem como dos níveis iniciais de força muscular.

A realização do TF em indivíduos jovens pode ajudar a fortalecer as estruturas ósseas, facilitar o controle de massa corporal, diminuir os agentes de risco cardiovascular e aumentar as habilidades motoras e o desempenho esportivo, diminuindo o risco de lesão nesse tipo de atividade e nas atividades recreacionais. ${ }^{15}$ Os potenciais benefícios do TF para jovens estão sintetizados no quadro abaixo:

Quadro 1 - Potenciais benefícios do treino de força em jovens

\begin{tabular}{l}
\hline Aumento força muscular \\
Aumento da potência muscular \\
Aumento da resistência muscular \\
Aumento da capacidade cardiorrespiratória \\
Melhora do perfil lipídico \\
Melhora composição corporal \\
Melhora das habilidades motoras \\
Aumento da performance desportiva \\
Aumento da resistência às lesões \\
\hline Fonte: Adaptado de FAIGENBAUM.
\end{tabular}

\section{Recomendações}

A adição do TF às crianças e adolescentes atletas deveria ser aplicada de forma cuidadosa pela repetição crônica e pelo estresse imposto ao sistema musculoesquelético dos indivíduos em questão, especialmente algumas crianças com sinais de imaturidade no sistema 
musculoesquelético, já que essas não serão tão hábeis para tolerar a mesma quantidade de exercícios que a maioria dos seus colegas de treino consegue tolerar. Sua individualidade biológica resulta em síndromes de estresses manifestadas por uma variedade de condições, incluindo tendinites e fraturas por estresse.

Assim sendo, programas de TF não deveriam ser simplesmente adicionados à rotina de exercícios, que talvez já incluam várias horas de brincadeiras e treinamentos específicos. Preferivelmente, o TF para crianças e adolescentes deveria ser incorporado em um programa de condicionamento periodizado que variasse em volume e intensidade ao longo do ano. ${ }^{24}$

Além disso, alguns fatores de risco como desequilíbrio muscular, rigidez muscular e articular, e pouca condição física, deveriam ser identificados para que os treinadores possam conhecer as necessidades específicas de cada criança. Em algumas instâncias talvez, torna-se necessário para alguns jovens atletas reduzir seu envolvimento desportivo, visando obter mais tempo para conseguir uma condição preparatória adequada de tal modalidade em questão.

Almejando começar um TF, a criança deve estar mental e emocionalmente pronta para consentir com as instruções do técnico e se submeter ao estresse do programa de treinamento que deve levar em conta o nível físico, emocional e maturacional da criança. Os instrutores devem reconhecer essas diferenças maturacionais e estarem cientes das predisposições genéticas dos alunos. As crianças não devem ser consideradas como adultos em miniatura, por isso os pressupostos e filosofias de treino dos mais velhos, não devem ser impostos às crianças. ${ }^{24}$ Para que o TF seja seguro e eficaz, algumas linhas de orientação são, portanto, recomendadas para a sua aplicação em crianças. 


\section{Linhas de orientação para o treinamento de força em crianças e adolescentes}

Como visto anteriormente, algumas informações devem ser aconselhadas: ${ }^{4,15,24}$

1. Adaptar o programa de treinamento ao nível de desenvolvimento psicológico do participante: a aderência a programas de treinamento requer uma maturidade emocional e a habilidade de seguir instruções. Essas características infelizmente não são possuídas por todas as crianças. Já em adolescentes, por sua vez, o interesse em TF torna-se um pouco maior e a habilidade de tolerar programas de treinamento já é considerável.

2. Instrução dirigida por profissionais qualificados: programas de treinamento devem ser conduzidos por instrutores, professores e técnicos qualificados que não só entendam os princípios básicos do TF, mas também compreendam a singularidade da infância. Supervisão minuciosa, instrução adequada à idade, e um ambiente seguro para os exercícios são requisitos fundamentais. Ademais, a realização de um exame médico prévio não é necessária para crianças aparentemente saudáveis.

3. Começar gradualmente a carga de treino e progressivamente aumentá-la: tem sido recomendado que crianças e adolescentes treinem de duas a três vezes por semana em dias não consecutivos e realizem de uma a três series de seis a quinze repetições em uma variedade de exercícios que foquem grandes grupos musculares. Todavia, no começo do programa de treinamento, começar com séries únicas de dez a quinze repetições por 
exercício, duas vezes por semana. Isso não só permitirá mudanças no desempenho muscular, mas irá prover uma oportunidade dos participantes ganharem confiança em suas habilidades antes de progredir para níveis mais elevados (Quadro 2, linhas de orientação para o TF em crianças e adolescentes). Além disso, ganhos contínuos podem ser feitos por aumentos graduais da carga, do número de repetições, ou pelo número de séries. Na média, um aumento de cinco a dez por cento na carga de treino é apropriado para a maioria dos exercícios. Uma vez que o número desejado de repetições possa ser feito, a carga pode ser aumentada de forma gradual, e as repetições podem ser diminuídas para permitir ganhos contínuos. Isso não significa que toda a sessão de treino necessita ser mais intensa que a sessão prévia; todavia a demanda sobre o indivíduo deveria ser gradualmente aumentada ao longo do tempo.

4. Aderência aos princípios de treinamento: carga apropriada, progressão gradual, e recuperação adequada entre as sessões de exercício são considerações importantes. Muito frequentemente, o volume e a intensidade do TF excedem as capacidades dos participantes e os períodos de descanso são muito curtos para uma recuperação adequada. Esse método pode ser particularmente perigoso para crianças porque não só aumenta o risco de lesão, mas também enfraquece o divertimento decorrente do programa de treinamento. Apesar de ser tentador seguir um programa de treinamento das academias ou um treino descrito por profissionais de saúde, cada participante deve ser tratado 
individualmente, em que a intensidade, o volume e a progressão do treino devem ser cuidadosamente prescritos.

5. Técnicas apropriadas devem ser ensinadas e reforçadas: apesar de alguns participantes quererem testar quanto de carga máxima conseguem levantar no primeiro dia de programa, o seu interesse e entusiasmo no TF devem ser direcionados ao desenvolvimento apropriado da forma e da técnica de exercício (controlar o movimento e respiração adequada, por exemplo).

6. Enfatizar divertimento intrínseco: em programas de TF o divertimento, a melhora das habilidades e o sucesso pessoal são fatores que devem estar evidentes. $\mathrm{O}$ uso de registros personalizados em cada sessão pode auxiliar na melhora de força de cada participante, e fazer com que acompanhem o desenvolvimento.

7. Incorporar variedades no programa de treinamento: adicionar exercícios novos, alterando o modo de treino, e variando o número de séries e repetições podem ajudar a manter o programa "atraente" e desafiador. Incorporar exercícios calistênicos, bandas elásticas e bolas com pesos diversos no TF podem ser efetivos, benéficos e divertidos.

8. Considerar múltiplos objetivos: os objetivos de um programa de TF não devem se limitar apenas à melhora da força muscular. O treinamento deve propor conhecimento sobre o corpo, promover procedimentos de 
treinamento seguro e ser um estímulo para que o participante tenha uma atitude mais positiva sobre o TF e a atividade física.

Quadro 2 - Linhas de orientação

\begin{abstract}
Prover instrução e supervisão adequada
Assegurar um ambiente de treino seguro

Ensinar os benefícios e os riscos associados ao TF

Iniciar a sessão de treino com aquecimento de 5 a 10 minutos

Começar com uma série leve de 10 a 15 repetições em exercícios

Focar na técnica correta ao invés da carga a ser levantada

Realizar TF duas a três vezes por semana em dias não consecutivos

Manter o programa de treino "atraente" e desafiador, variando-o continuamente
\end{abstract}

Fonte: Adaptado de FAIGENBAUM. ${ }^{15}$

\title{
CONCLUSÃO
}

Muitos são os benefícios relacionados à saúde e à qualidade de vida adquiridos por crianças e adolescentes que participam de programas que englobem TF dentro de suas atividades. Todavia, estes benefícios somente são constatados quando as atividades são conduzidas por instrutores competentes, que conheçam os princípios e o desenvolvimento dos programas de TF.

Apesar de haver alguns riscos e algumas limitações acerca do conhecimento que rege a prática do TF específico para crianças, já é possível ter consciência de que os benefícios fisiológicos, biomecânicos e até mesmo sociais recebem uma maior importância neste processo. Cabe ao professor ter consciência que estas melhorias estão subordinadas a uma prática adequada de um método de treino em conformidade com a faixa etária e receptividade da criança. Da mesma forma, o professor deve ter o conhecimento de que o TF pode ser seguro e eficaz para crianças, mas que não é necessário para toda e qualquer criança. 
Em que pese os assuntos discutidos anteriormente, as evidências científicas indicam que o TF pode ser saudável e apresentar benefícios para crianças e adolescentes quando esses forem instruídos apropriadamente por instrutores qualificados que se baseiem em linhas de orientação corretas de treino. Assim sendo, acredita-se que as recomendações compiladas dentre os artigos revisados permitem que o professor de Educação Física tenha um mínimo embasamento teórico-prático na prescrição do TF para crianças e adolescentes.

\section{REFERÊNCIAS}

${ }^{1}$ FAIGENBAUM, A. D. Resistance training among young athletes: safety, efficacy and injury prevention effects. British Journal of Sports Medicine, v. 44, n. 4, p. 56-63, abr. 2010.

${ }^{2}$ COUTTS, A.; MURPHY, A.; DASCOMBE, B. Effect of direct supervision of a strength coach on measures of muscular strength and power in young rugby league players. Journal of Strength and Conditioning Research, v.18, n.2, p.316-323, mar. 2004.

${ }^{3}$ OLSEN, O.; MYKLEBUST, G.; ENGEBRETSEN, L. Exercises to prevent lower limb injuries in youth sports: cluster randomised controlled trial. British Medical Journal, v. 26, n. 2, p.330-449, fev. 2005.

${ }^{4}$ FAIGENBAUM, A. D. et al. Youth resistance training: updated position statement paper from the National Strength and Conditioning Association. Journal of Strength and Conditioning Research, v. 23, n. 5, p. 60-79, jan. 2009. 
${ }^{5}$ BLIMKIE, C. J. Resistance training during preadolescence: issues and controversies. Sports Medicine, v.15, p.389-407, jun. 1993.

\section{${ }^{6}$ AMERICAN ACADEMY OF PEDIATRICS COUNCIL ON SPORTS MEDICINE AND} FITNESS. Strength training by children and adolescents. Pediatrics, v.121, n. 4, p. 835-840, abr. 2008.

${ }^{7}$ HAMILL, B. Relative safety of weightlifting and weight training. Journal of Strength and Conditioning Research, v. 8, n.1, p. 53-57, out. 1994.

${ }^{8}$ CENTERS FOR DISEASE CONTROL AND PREVENTION. Sports-related injuries among high school athletes: United States, 2005-06 school year. Morbidity and Mortality Weekly Report, v. 55, n.38, p.1037-1040, set. 2006.

${ }^{9}$ MARGINSON, V. et al.Comparison of the symptoms of exercise-induced muscle damage after an initial and repeated bout of plyometric exercise in men and boys. Journal Applied of Physiology, v. 99, n.5, p.1174-1181, abr. 2005.

${ }^{10}$ CLARKSON, P. M. Case report of exertional rhabdomyolysis in a 12-year-old boy.

Medicine and Science in Sports and Exercise, v.38, n.2, p.197-200, mar. 2006.

${ }^{11}$ KERR, Z.Y., COLLINS, C. L., COMSTOCK, R. D. Epidemiology of weight trainingrelated injuries presenting to United States Emergency Departments, 1990 to 2007. American Journal of Sports Medicine, v. 38, n.7, p.765-771, mar. 2010. 
${ }^{12}$ LILLEGARD W. A.; BROWN E.W.; WILSON D. J. Efficacy of strength training in prepubescent to early postpubescent males and females: effects of gender and maturity. Pediatric Rehabilitation, v.1, n.3, p.147-157, fev. 1997.

${ }^{13}$ FAIGENBAUM, A. D.; MILLIKEN L. A.; LOUD R. L. Comparison of 1 and 2 days per week of strength training in children. Research Quarterly for Exercise and Sport, Estados Unidos da América, v.73, n. 4, p.416-424, dez. 2002.

14 FAIGENBAUM A. D.; MILLIKEN L. A.; MOULTON L. Early muscular fitness adaptations in children in response to two different resistance training regimens. Pediatric Rehabilitation, v.17, n.3, p.237-248, ago. 2005.

${ }^{15}$ FAIGENBAUM, A.D.; MYER, G.D. Youth resistance training. President's Council on Physical Fitness and Sports Research Digest, v. 4, n. 3, p.1-8, set. 2003.

${ }^{16}$ CAINE, D.; MAFFULLI, N.; CAINE, C. Epidemiology of Injury in Child and Adolescent Sports: Injury Rates, Risk Factors, and Prevention. Clinical Journal of Sport Medicine, v. 27, n.1, p.19-50, jan. 2008.

${ }^{17}$ MICHELI, L.; GLASSMAN, R.; KLEIN, M. The prevention of sports injuries in children. Clinical Journal of Sport Medicine, v.19, n.5, p.821-834, ago. 2000.

${ }^{18}$ FAIGENBAUM, A. et al. Youth Resistance training: position statement paper and literature review. Strength and Conditioning Journal, v.18, n.6, p. 62-75, nov. 1996. 
${ }^{19}$ BENSON, A.C.; TORODE, M.E.; FIATARONE, M. A. Effects of resistance training on metabolic fitness in children and adolescents: a systematic review. Obesity Reviews, v.9, n.1, p. 43-66, jan. 2008.

${ }^{20}$ LAU, P.W.C. et al. Effects of short: term resistance traning on serum leptin levelsin obese adolescents. Journal of Exercise Science and Fitness, v. 8, n.1, p. 54-60, mar. 2010.

${ }^{21}$ MORRIS, F.; NAUGHTON, G.; GIBBS, J. Prospective ten-month exercise intervention in premenarcheal girls: positive effects on bone and lean mass. Journal of Bone and Mineral Research, v.12, n. 9, p.1453-1462, set.1997.

${ }^{22}$ IGNJATOVIC, A.; RADOVANOVIC, D.; STANKOVIC, R. Influence of strength training program on isometric muscle strength in young athletes. Acta Medica Medianae. v. 46, n.1, p.16-20, jan. 2007.

${ }^{23}$ FAIGENBAUM, A. D. Strenght training for children and adolescents. Clinics in Sports Medicine, v.19, n.4, p.593-618, set. 2000.

${ }^{24}$ BRAGA, F. et al. Benefícios do treinamento de força para crianças e adolescentes escolares. Disponível em: <http://www.efdeportes.com/efd119/treinamento-de-forca.htm>. Acesso em: 12 set. 2010 . 